\title{
Wettability Control of Droplet Deposition and Detachment
}

\author{
Jean-Christophe Baret ${ }^{1, *}$ and Martin Brinkmann ${ }^{2, \dagger}$ \\ ${ }^{1}$ Philips Research Laboratories Eindhoven, Prof. Holstlaan 4, 5656 AA Eindhoven, The Netherlands \\ ${ }^{2}$ Biological Nanosystems Group, Interdisciplinary Research Institute c/o IEMN, Avenue Poincaré BP 69, \\ F-59652 Villeneuve d'Ascq, France
}

(Received 6 November 2005; published 14 April 2006)

\begin{abstract}
The conditions for droplet deposition on plane substrates are studied using electrowetting to continuously modulate the surface wettability. Droplets of controlled volume attached to the tip of a pipette are brought into contact with the surface. During retraction of the pipette the droplets are deposited or detach completely depending on volume and contact angle. The experimental limit of deposition in the contact angle or volume plane is in good agreement with analytical and numerical predictions obtained within the capillary model.
\end{abstract}

DOI: 10.1103/PhysRevLett.96.146106

PACS numbers: 68.05. $-\mathrm{n}, 68.08 . \mathrm{Bc}, 83.60 . \mathrm{Np}$

Small liquid droplets adhere to a solid substrate simply by a gain of interfacial energy. On superhydrophobic surfaces only small parts of the substrate establish contact with the liquid [1] and droplets may bounce back from the surface if their impact velocity is too high [2]. One can easily imagine that a poor wettability of the substrate makes it nearly impossible to detach small droplets from the tip of a pipette and to place them on the surface: if the adhesion energy is smaller than the work required to break the liquid at the tip, the droplet remains attached to the pipette. Inspired by this phenomenon we performed an experimental and theoretical study to quantify the effect of surface wettability and droplet volume on the mode of droplet instability. The series of images in Figs. 1(a) and 1(b) illustrate a complete redetachment and a successful deposition, respectively, of a small water droplet while retracting the pipette. Interestingly, analogous shape instabilities have been reported for fluid membranes, i.e., necking instabilities of stretched membrane tubes [3] or vesicle desorption from rigid substrates under the influence of a point force both for weak [4] and strong [5] membrane adhesion. However, for the capillary problem the mode of droplet instability has not been systematically studied. In this Letter, we will give a quantitative criterion for droplet detachment based on a stability analysis within the capillary model which is in perfect agreement with the experimental data. Our criterion can be directly applied in technological processes which involve the production and placement of small droplets, e.g., in spotting arrays of biomolecules [1,6] or in microcontact printing [7].

In our experimental setup, we use the electrowetting effect on dielectric substrates [8,9] as a convenient way to continuously modulate the contact angle of a conductive droplet with an applied ac voltage. In particular, we employed $n$-doped As conductive silicon wafers (Wacker Siltronics AG) coated with a silicon oxide insulating layer (thickness $T \approx 1.1 \mu \mathrm{m}$, dielectric constant $\varepsilon_{0} \varepsilon_{r}=$ $35 \mathrm{pF} / \mathrm{m}$ ) and hydrophobized with an octadecyltrichlorosilane (OTS) monolayer. Salt was added to the water $(\mathrm{NaCl}$
$0.3 \%$ mass concentration) in order to increase its electric conductivity. Silicon oil (Fluka DC200) was used as an ambient phase to suppress evaporation, decrease contact angle hysteresis to a value $\approx 2^{\circ}$, and to reduce the effect of gravity. With a density excess $\Delta \rho \approx 60 \mathrm{~kg} / \mathrm{m}^{3}$ of water over oil and a surface tension of the water-oil interface $\gamma=$ $(38 \pm 1) \mathrm{mN} / \mathrm{m}$, as measured by the pendant drop method
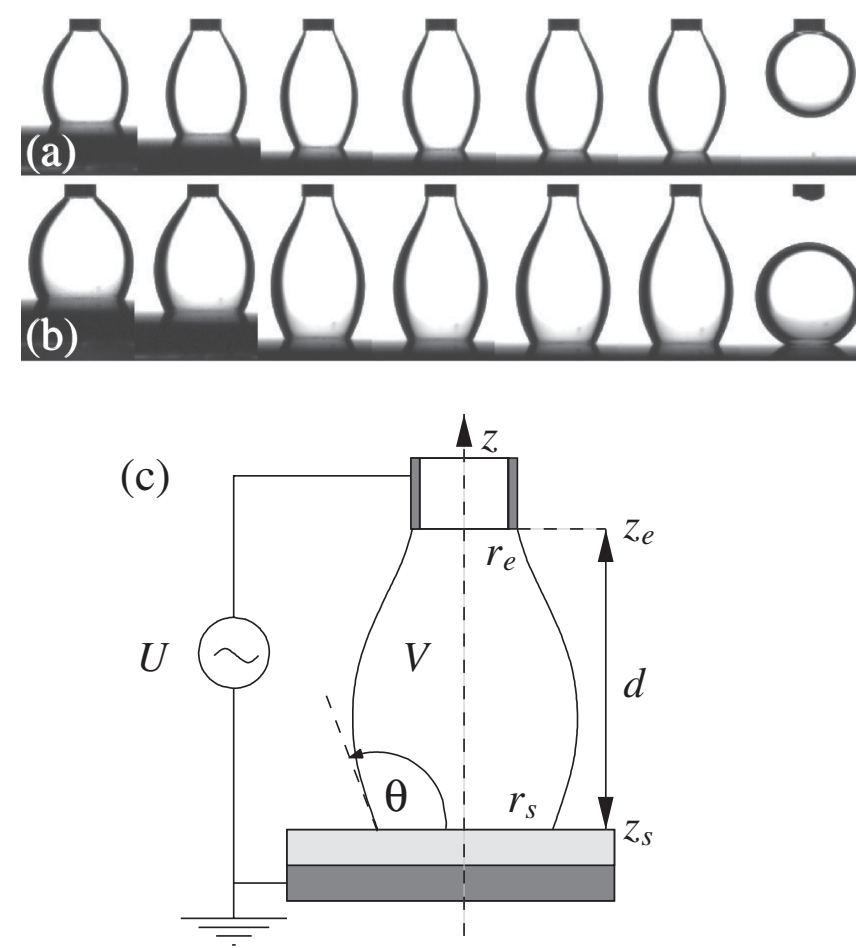

FIG. 1. Two typical sequences of detachment (a) and deposition (b) when the distance $d$ between substrate and pipette tip is quasistatically increased. The contact angle is $\theta=(118 \pm 2)^{\circ}$ in both cases. The volumes are $13 \mu \mathrm{L}$ (a) and $21 \mu \mathrm{L}$ (b) while the outer radius $r_{e}$ of the cylindrical pipette is $255 \mu \mathrm{m}$. (c) Sketch of the electrowetting setup. The Si-As substrate is covered by an hydrophobized $\mathrm{SiO}_{2}$ insulating layer. 
(Dataphysics-OCA30), the capillary length is $L_{c}=$ $(\gamma / \Delta \rho g)^{1 / 2}=8 \mathrm{~mm}(g$ being the acceleration of gravity). Droplets are extruded from a steel pipette (EFD-precision stainless tips, external radius $r_{e}=255 \mu \mathrm{m}$, internal radius $125 \mu \mathrm{m}$ ) used as a counterelectrode for the voltage supply; see Fig. 1(c). An ac voltage $U$ (frequency $f=$ $10 \mathrm{kHz}$ ) is applied between the electrode and the silicon wafer. Then, the substrate is approached to the droplet of fixed volume $V$ located at the tip of the pipette until a contact is established: the droplet spreads on the surface until the apparent contact angle $\theta$ reaches a voltage dependent equilibrium. The contact angle measured from the side is in good agreement with Lippmann's equation

$$
\cos \theta=\cos \theta_{Y}+\frac{\varepsilon_{0} \varepsilon_{r}}{2 T \gamma} U^{2}
$$

up to $50 \mathrm{~V}$. Here, $\theta_{Y}=155^{\circ}$ is Young's contact angle of the droplet on the substrate, i.e., at zero voltage. Above $50 \mathrm{~V}$ saturation effects are observed [9]. We restrict our analysis to the regime where Lippmann's equation holds, i.e., $U<50 \mathrm{~V}$, which allows a contact angle modulation in the range $90^{\circ}<\theta<155^{\circ}$.

The distance $d$ between the tip of the pipette and the substrate is then increased in steps of $\delta=10 \mu \mathrm{m}$ and corresponding images of the droplet at equilibrium are registered. The radius of the droplet-substrate interface $r_{s}$ and the tip-substrate distance $d$ are extracted by image processing. As shown in Fig. 1, droplets elongate during an increase of the distance $d$ while the radius $r_{s}$ of the contact line decreases and the contact angle $\theta$ on the substrate remains constant within $2^{\circ}$. The range of locally stable droplets bridging the substrate and tip is limited by a maximal distance $d_{\max }$. Having reached $d=d_{\max }$ two situations are observed: the droplet either detaches from the substrate (regime called "detachment") or breaks away from the pipette which leads to a deposited droplet (regime called "deposition").

The transition between the two regimes is studied for a variety of fixed contact angles by changing the droplet volume. At given contact angle a droplet is deposited at volumes $V$ larger than a critical value $V_{c}$ while detachment occurs for $V<V_{c}$. The experimental results are displayed in Fig. 2 showing that $V_{c}$ increases with increasing $\theta$ : the poorer the wettability of the surface the larger the minimal droplet volume leading to deposition. The experimentally determined points compare well to the predictions obtained within the capillary model; cf. Fig. 2. At fixed volume the regions of detachment and deposition are limited by a critical contact angle $\theta_{c}$.

A theoretical analysis of the droplet instabilities has been performed in the capillary model and for axisymmetric droplet configurations. As the electric field is confined to a very small region around the contact line [9], the Maxwell stress solely enters the effective droplet-substrate surface tension and, assuming neutral buoyancy, we can consider the liquid-liquid interface as a surface of constant

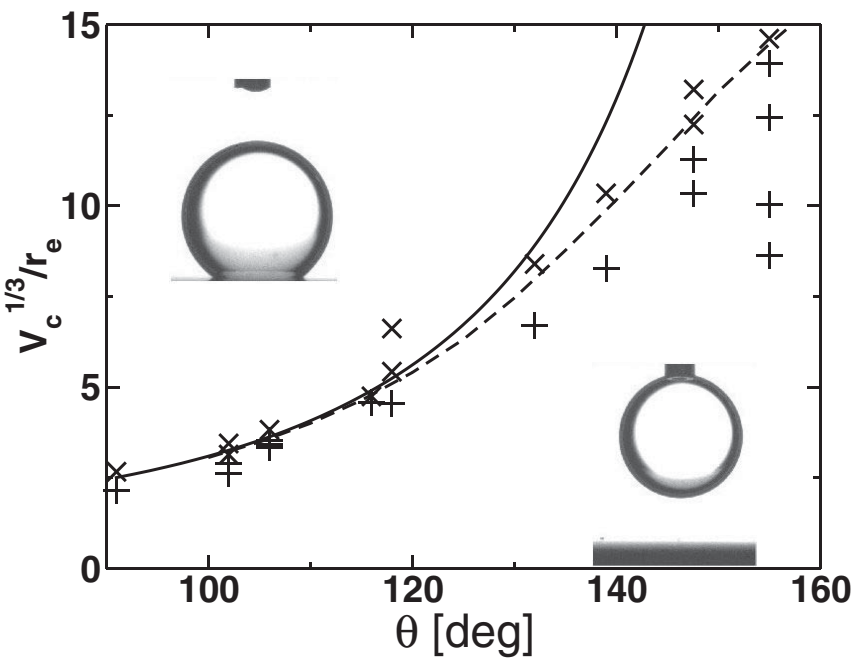

FIG. 2. Deposition and detachment regime in the $(\theta, V)$ diagram. The full line refers the analytical expression given by Eq. (4). For large volumes the influence of gravity on the limit between deposition $(X)$ and detachment $(+)$ is obtained by numerical minimization (dashed line) using the program Surface Evolver [16] for a capillary length $L_{c} / r_{e}=32$.

mean curvature. To determine local stability of a bridging droplet we have to consider the complete set of stationary droplet shapes for a given tip-substrate distance. It is reasonable to assume that the liquid bridges are axisymmetric surfaces close to breakup. In principle, all axisymmetric bridging shapes are parametrized by Delaunay surfaces [10] and among them we only consider stretched solutions corresponding to unduloids [11]. The radius of the unduloid is parametrized in cylindrical coordinates by a function $r\left(r_{b}, r_{n}, z\right)$ involving elliptic integrals of the first and second kind [12]. The parameters $r_{b}$ and $r_{n}$ of the unduloid correspond to the maximal (bulge) and minimal (neck) radius of the shape, respectively, while $z$ is the coordinate on the symmetry axis. Stationary shapes are given by unduloid segments which satisfy four subsidiary conditions given by the tip radius $r_{e}$ of the pipette, the contact angle $\theta$ on the substrate, the droplet volume $V$, and the tip-substrate distance $d$ :

$$
\left.\begin{array}{ccc}
r\left(r_{b}, r_{n}, z_{e}\right) & = & r_{e} \\
\left.\frac{d r\left(r_{b}, r_{n}, z\right)}{d z}\right|_{z=z_{s}} & = & \cot \theta \\
\int_{z_{s}}^{z_{e}} \pi r^{2}\left(r_{b}, r_{n}, z\right) d z & = & V \\
z_{e}-z_{s} & = & d .
\end{array}\right\}
$$

The system of Eqs. (2) may have several solutions for a given set of parameter values $\left(r_{e}, \theta, V, d\right)$. Each solution corresponds to either a local minimum (or even global minimum) or a saddle point in the energy landscape.

The first condition is used to determine the position $z_{e}$ of the pipette tip. As illustrated in Fig. 3(a) one has two solutions $z_{e}^{+}$and $z_{e}^{-}$or none. The second condition of a given contact angle on the substrate is fulfilled either at one of two positions $z_{s}^{+}$and $z_{s}^{-}$, enclosing an inflection point $P_{\mathrm{i}}$, or nowhere, see Fig. 3(a). The number of possible 


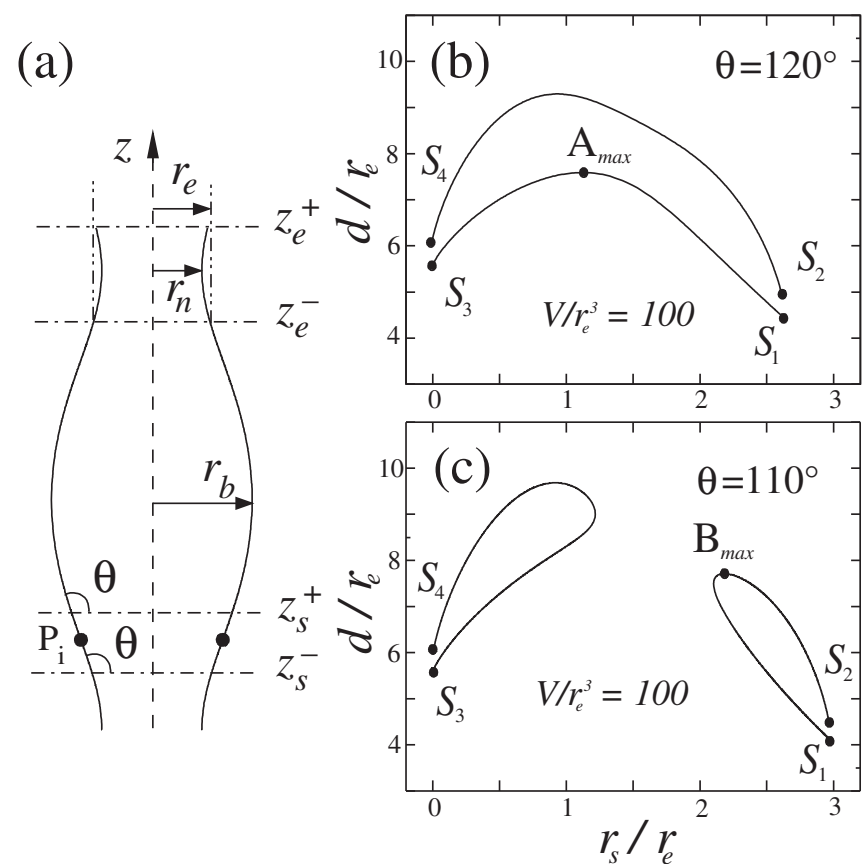

FIG. 3. (a) Sketch of an unduloid with positions $z_{s}^{ \pm}$and $z_{e}^{ \pm}$ indicated where the respective subsidiary conditions of a given contact angle and a contact line pinned at the tip of the pipette are satisfied. The dots indicate the position of the inflection point $P_{\mathrm{i}}$. (b) Branches of stationary solutions. The spherical limiting shapes $\mathcal{S}_{1}$ and $\mathcal{S}_{3}$ are connected (regime I). (c) $\mathcal{S}_{1}$ connected to $\mathcal{S}_{2}$ (regime II). According to (4) we find a contact angle $\theta=$ $114.25^{\circ}$ at the transition of the branch topology for $V / r_{e}^{3}=100$.

solution classes can be reduced since segments longer than one period are always found unstable $[13,14]$. Segments which lack a maximum turn out to be irrelevant to our analysis in the case of hydrophobic contact angles $\theta>$ $\pi / 2$. Finally, four different classes of solutions are left to be considered: unduloid shapes $(1, \ldots, 4)$ generated by plane cuts at $\left\{z_{e}^{-}, z_{s}^{+}\right\},\left\{z_{e}^{+}, z_{s}^{+}\right\},\left\{z_{e}^{-}, z_{s}^{-}\right\}$, and $\left\{z_{e}^{+}, z_{s}^{-}\right\}$, respectively. In the limit $r_{n} \rightarrow 0$ the unduloid surface tends to a chain of kissing spheres and, hence, to each class of solutions $(i=1, \ldots, 4)$ corresponds a particular spherical limiting shape $\mathcal{S}_{i}$. A singular neck appears close to the tip while approaching shapes $\mathcal{S}_{2}$ or $\mathcal{S}_{4}$. The limiting shapes $\mathcal{S}_{3}$ and $\mathcal{S}_{4}$ exhibit a singular neck at the substrate. Solutions beyond the regular shape $\mathcal{S}_{1}$ are nodoids $[10,12]$, the second class of Delaunay surfaces.

In order to numerically solve the system of Eqs. (2), we fix the parameters $\left(r_{e}, \theta, V\right)$, leaving the distance $d$ free and search for the solution in each class of unduloid shapes. The solutions are parametrized by the radius $r_{s}$ which acts as an "order" parameter. This arbitrary choice of representation has the advantage that both $r_{s}$ and $d$ are easily accessible in our experiments. The Figs. 3(b) and 3(c) illustrate the calculation at a volume fixed to $V / r_{e}^{3}=$ 100. The solutions are represented in a $\left(r_{s}, d\right)$ diagram where they form continuous branches. Depending on the value of $\theta$ two regimes are observed.
In regime I we find two solution branches, one connecting the limiting shape $\mathcal{S}_{1}$ with $\mathcal{S}_{3}$, and a second connecting $\mathcal{S}_{2}$ with $\mathcal{S}_{4}$; cf. Fig. 3(b). Solutions on the branch starting from $\mathcal{S}_{1}$ are locally stable up to the turning point $A_{\max }$. In regime II, however, the branches form two distinct lobes which connect $\mathcal{S}_{1}$ and $\mathcal{S}_{2}$ as well as $\mathcal{S}_{3}$ and $\mathcal{S}_{4}$; see Fig. 3(c). Following the solution branch from $\mathcal{S}_{1}$ to larger distances we arrive at a turning point $B_{\max }$ : no locally stable solution is found at higher distances.

Figure 4 shows the comparison between the model for $\theta=101^{\circ}$ and the experimental measurements of the parameters $\left(r_{s}, d\right)$ for deposition (down triangles) and detachment (up triangles) at $\theta=(101 \pm 1)^{\circ}$ and $V^{1 / 3} / r_{e}=$ $2.6,2.9,3.1,3.4$. The two modes of instability can be distinguished by the evolution of the radius $r_{s}$ as the distance $d$ is increased. When $d$ approaches $d_{\max }$, the radius $r_{s}$ displays a short increase before the instability sets in (deposition regime or regime II), or decreases rapidly ere the droplet detaches from the substrate (detachment regime or regime I). Hence, the experimental detachment and deposition regimes are, respectively, correlated to the theoretical regimes I and II. Physically, while pulling, $r_{s}$ shrinks which reduces the adhesion force. In regime I, the pulling force ultimately overcomes adhesion leading to detachment. In regime II, at large volume or wettability, the larger adhesion force ties the droplet to the substrate. Instead of detaching, the droplet develops a neck close the tip before the liquid breaks.

An accurate approximation of the droplet shape at the transition between regime I and II is given by an unduloid

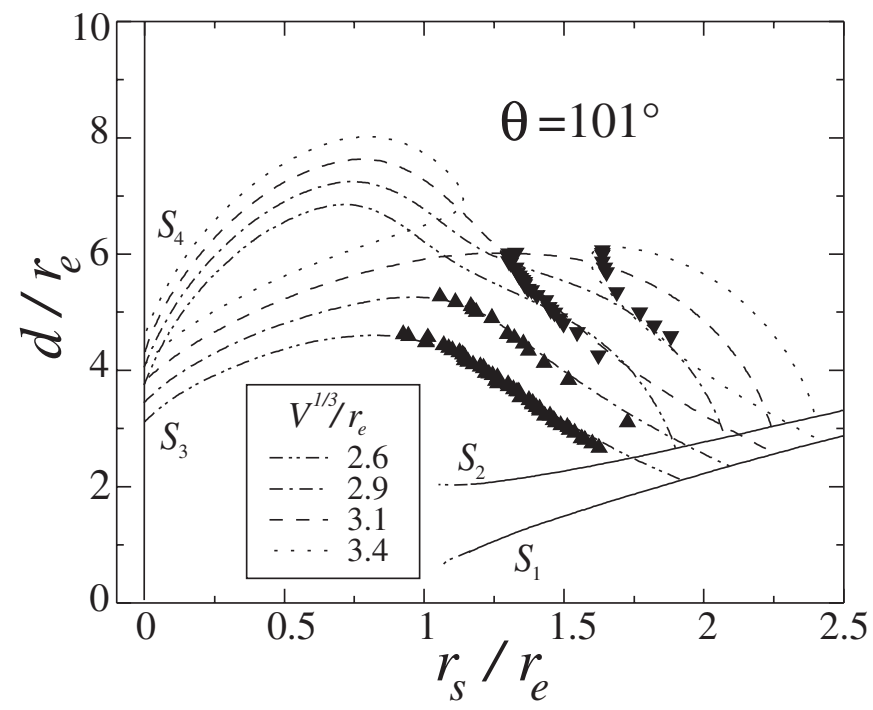

FIG. 4. Solution branches in the $\left(r_{s}, d\right)$ diagram showing the two possible scenarios of instability. Experimentally observed deposition (down triangles) corresponds to regime II while detachment (up triangles) corresponds to regime I. The broken curves illustrate the theoretical predictions for different droplet volumes and terminate on a branch of spherical limiting shapes $\mathcal{S}_{i}$ (full lines). 
segment where all classes (1-4) meet, i.e., with an inflection point on the substrate and a neck radius which equals the radius of the tip. In the $\left(r_{s}, d\right)$ diagram, it appears on the left, unstable lobe in regime II and at a droplet volume very close to the transition. The boundary conditions for this special unduloid segment read

$$
\left.\begin{array}{ccc}
r_{n}= & r_{e} \\
r_{b}= & (1-\cos \theta) /(1+\cos \theta) r_{e} \\
r_{s}= & \left(r_{b} r_{n}\right)^{1 / 2}
\end{array}\right\}
$$

and determine the estimated transition volume $V_{c}$ as a function of the contact angle $\theta$ :

$$
\begin{aligned}
\frac{3 V_{c}}{\pi r_{e}^{3}}= & \frac{\left(7+x^{2}\right)(1-x)}{(1+x)^{3}}\left[2 \mathcal{E}\left(k, \frac{\pi}{2}\right)-\mathcal{E}\left(k, \frac{\theta}{2}\right)\right] \\
& -\frac{(1-x)}{(1+x)}\left[2 \mathcal{F}\left(k, \frac{\pi}{2}\right)-\mathcal{F}\left(k, \frac{\theta}{2}\right)\right]-\frac{16 x}{(1+x)^{3}} .
\end{aligned}
$$

Here, we use the abbreviation $x=\cos \theta$ and the elliptic integrals of the first and second kind

$$
\left.\begin{array}{l}
\mathcal{E}(k, \varphi)=\int_{0}^{\varphi} d \psi\left(1-k^{2} \sin ^{2} \psi\right)^{1 / 2} \\
\mathcal{F}(k, \varphi)=\int_{0}^{\varphi} d \psi\left(1-k^{2} \sin ^{2} \psi\right)^{-1 / 2}
\end{array}\right\},
$$

respectively, with a modulus $k^{2}=-4 x /(1-x)^{2}$.

Inserting a contact angle of $\theta=(114.25 \pm 0.05)^{\circ}$ into (4) gives a volume of $V_{c} / r_{e}^{3}=100 \pm 0.6$, in excellent agreement with the experimental values. The transition volume $V_{c}$ according to expression (4) is displayed as the full line in Fig. 2 and matches with the experimentally determined limit up to $\theta=120^{\circ}$. For larger contact angles, a mismatch is observed due to the influence of gravity. In the limit $\theta=180^{\circ}$, adhesion vanishes and our model predicts a diverging volume $V_{c}$. In contrast, the volume for gravity induced deposition ("dripping faucet") scales as $V_{g} \propto L_{c}^{2} \times r_{e}$ [15]. The limit between deposition and detachment displays a transition from the volume $V_{c} \propto$ $f(\theta) r_{e}^{3}$ with the expression $f(\theta)$ on the right-hand side of Eq. (4) to $V_{g}$ as $\theta$ tends to $180^{\circ}$. This defines a capillary length $L_{c}^{*} \propto[f(\theta)]^{1 / 2} r_{e}$ at the crossover between adhesion dominated and gravity dominated deposition. The influence of gravity is numerically confirmed by minimizations with the software Surface Evolver [16] using the experimental value $L_{c} / r_{e}=32$. As in our experiments we increase the distance in small steps, we minimize the interfacial energy for each value of $d$ and record the evolution of $r_{s}$. Qualitatively, the evolution of $r_{s}$ is identical to the scenario found in the analysis for zero gravity. Further experiments performed at $L_{c} / r_{e}=10$ show that gravity influences significantly the deposition already at $\theta \lesssim 100^{\circ}$.

In summary, we have studied experimentally the transition between droplet deposition and detachment on surfaces of various wettabilities. It is demonstrated that electrowetting can be used as a convenient and robust tool to achieve an active control of surface wettability. A clear transition between deposition and detachment occurs at a critical volume which is a monotonously increasing function of the contact angle. If gravity is negligible, an estimate of the transition volume is given in terms of an analytical expression and in quantitative agreement with the experimental data. For the particular system considered here, the analysis of droplet instabilities can be based on the evolution of mechanical equilibrium states and does not evoke the dynamics of breakup [17] or contact line motion.

Our experimental setup can be combined with the "pin technique" used in microarray production [6]: if the substrate is equipped with a pattern of electrodes one may obtain a full control on droplet deposition by applying a voltage during liquid contact. The electrode pattern guarantees deposition on the selected location.

The authors thank Michel Decré, Ralf Blossey, and Frieder Mugele for their support and Emile van Thiel, Anton Kemmeren, and Dirk Burdinski for technical assistance. J.-C. Baret acknowledges financial support by a Marie Curie Industry Host Fellowship IST-1999-80004.

*Electronic address: jc.baret@isis.u-strasbg.fr Present address: ISIS-ULP, Laboratoire de Biologie Chimique, 8, alle Gaspard Monge, 67083 Strasbourg (F). †Electronic address: martin.brinkmann@ds.mpg.de Present address: Max-Planck-Institute for Dynamics and Self-Organization, Bunsenstr. 10, 37073 Göttingen (D).

[1] R. Blossey, Nat. Mater. 2, 301 (2003).

[2] D. Richard, C. Clanet, and D. Queré, Nature (London) 417, 811 (2002).

[3] V. A. Frolov et al., Proc. Natl. Acad. Sci. U.S.A. 100, 8698 (2003).

[4] A. S. Smith, E. Sackmann, and U. Seifert, Phys. Rev. Lett. 92, 208101 (2004).

[5] A. S. Smith, E. Sackmann, and U. Seifert, Europhys. Lett. 64, 281 (2003).

[6] P. Belaubre et al., Appl. Phys. Lett. 82, 3122 (2003).

[7] Y. Xia, K.E. Paul, J.A. Rogers, and G. M. Whitesides, Chem. Rev. 99, 1823 (1999).

[8] B. Berge, C. R. Acad. Sci. Paris, Ser. II 317, 157 (1993).

[9] F. Mugele and J.-C. Baret, J. Phys. Condens. Matter 17, R705 (2005).

[10] C. Delaunay, J. Math. Pures Appl. 6, 309 (1841).

[11] Nodoids and nonaxisymmetric shapes become relevant if the droplet is pushed into the direction of the substrate.

[12] R. J. Roe, J. Colloid Interface Sci. 50, 70 (1975).

[13] D. Langbein, Capillary Surfaces (Springer, New York, 2002).

[14] L. Rayleigh, Proc. R. Soc. London, Ser. A 29, 71 (1879).

[15] O. E. Yildirim, Q. Xu, and O. A. Basaran, Phys. Fluids 17, 062107 (2005).

[16] K. Brakke, Exp. Math. 1, 141 (1992).

[17] J.-C. Baret and F. Mugele, Phys. Rev. Lett. 96, 016106 (2006). 